\title{
Comparison of Vacuum Pressures and Forces Generated by Different Catheters and Pumps for Aspiration Thrombectomy in Acute Ischemic Stroke
}

\author{
Michael T. Froehler \\ Cerebrovascular Program, Vanderbilt University Medical Center, Nashville, TN, USA
}

\section{Keywords}

Aspiration thrombectomy · ADAPT · Stroke $\cdot$ Mechanical thrombectomy · Aspiration catheter

\begin{abstract}
Background: Direct aspiration thrombectomy is an increasingly utilized technique in endovascular stroke treatment that relies on vacuum and suction force to remove the clot. This report assesses the pressures and forces generated by different catheters and pumps. Methods: Vacuum pressures were measured using a vacuum gauge for several catheters (Stryker Catalyst6, Penumbra Ace 064, Medtronic Arc 061, and Penumbra 041) and pumps (Penumbra pump, $60-\mathrm{mL}$ syringe, and the ASPIRE device). Suction forces were calculated based on pressure and catheter tip size (force $=$ area $\times$ pressure). Vacuum pressures and forces were also assessed with a coaxial microcatheter (Rebar 18; inner diameter $=0.021$ inches), mimicking a combined aspiration and stent retriever approach. Results: All catheters transmitted similar vacuum pressures, but suction force was proportionate to catheter tip area. Pump vacuum pressures were also similar, although the Penumbra pump (mean -25.63 inches $\mathrm{Hg}$ [inHg]) was slightly weaker than the syringe and the ASPIRE device $(-27.04$ and $-27.58 \mathrm{inHg}$, respectively; $p<0.001$, two-way ANOVA). A coaxial microcatheter lowered the mean vacuum pressure by only $1.2 \mathrm{inHg}(p=0.005)$, though it would theoretically create a very significant reduction in suction force if partially blocking the aspiration catheter tip area. Conclusions: All catheters transmit similar vacuum pressure, but the suction force on the clot is stronger with larger catheter tips. Coaxial microcatheters will have a minimal impact on suction force as long as they are proximal to the aspiration catheter tip. Currently available thrombectomy suction devices, including the Penumbra pump, the ASPIRE handheld pump, and the $60-\mathrm{mL}$ syringe, all develop similar vacuum pressures.




\section{Introduction}

Thrombectomy for acute ischemic stroke due to large vessel occlusion has become the standard of care [1] and is being performed with increasing frequency around the world [2]. The endovascular techniques employed in thrombectomy continue to evolve rapidly and now include the use of multiple clot retrieval devices, balloon guide catheters, and local aspiration catheters [3-5]. One technique that has gained significant popularity is direct aspiration thrombectomy (A Direct Aspiration first-Pass Thrombectomy [ADAPT]), wherein a largebore catheter is navigated directly to the proximal face of the clot, vacuum is applied to the catheter hub, and the clot is then removed by pulling the catheter out while continuing suction [6-8].

The ADAPT technique is particularly compelling as it offers the possibility of more rapid recanalization $[9,10]$. However, this speed relies on the efficacy of the maneuver, which in turn relies on a reliable vacuum source (pump) and a highly navigable large-bore catheter. Ultimately, successful thrombectomy using this technique depends on a strong vacuum force between the tip of the catheter and the clot in order to extract it.

This static force between the catheter and the clot must be differentiated from the rate of flow that an individual system is capable of. Specifically, the rate of flow of a fluid through a catheter is governed by Poiseuille's law, which states that flow is a function of radius - to the fourth power, pressure, viscosity, and length. Others have examined the flow rates of various thrombectomy catheters $[11,12]$ and found that indeed the size of the catheter is the greatest determinant of flow. However, in direct aspiration thrombectomy, the catheter tip is purposefully occluded by the clot, and thus flow rate is irrelevant. Rather, in this circumstance the force exerted on the clot is simply a product of the vacuum pressure and the area of the catheter tip (F = AP).

Just as interventional techniques have evolved rapidly, so too have new devices been developed to enable these new approaches. In particular, several new large-bore catheters have been released that may have potential for direct aspiration thrombectomy in acute stroke. Furthermore, there are now several options for aspiration pumps. While the Penumbra system has offered an electrical pump since its inception [13], many interventionalists have opted for manual aspiration with a large syringe $[14,15]$. There is also the relatively new ASPIRE aspiration pump that has been used for peripheral thrombectomy [16] and consists of a handle-actuated, modified syringe with valves to allow for continuous vacuum generation while purging aspirated blood. This device could also prove useful in stroke treatment, given its ability to maintain a continuous aspiration force.

Finally, some users advocate a combined technique using a stent retriever and an aspiration catheter for thrombectomy in stroke $[17,18]$. When using such a coaxial approach, the stent retriever delivery microcatheter may partially occlude the aspiration catheter and thereby potentially reduce the vacuum force. Therefore, some have recommended a "barewire thrombectomy" technique wherein the delivery catheter is removed prior to aspiration and thrombectomy [19]. These authors have also assessed flow rates through the aspiration catheter with and without the microcatheter in place (Poiseuille fluid dynamics), but have not assessed the static suction force in this scenario.

In light of these developments and considerations, we aimed to assess vacuum pressure at the catheter tip in the newer generation of highly navigable catheters in combination with three aspiration pumps: the Penumbra pump, a 60-mL syringe, and the ASPIRE device. We also performed a substudy to determine the effect of a coaxial stent retriever delivery catheter on the suction force at the tip of the aspiration catheter. 
Froehler et al.: Comparison of Vacuum Pressures and Forces Generated by Different Catheters and Pumps for Aspiration Thrombectomy in Acute Ischemic Stroke
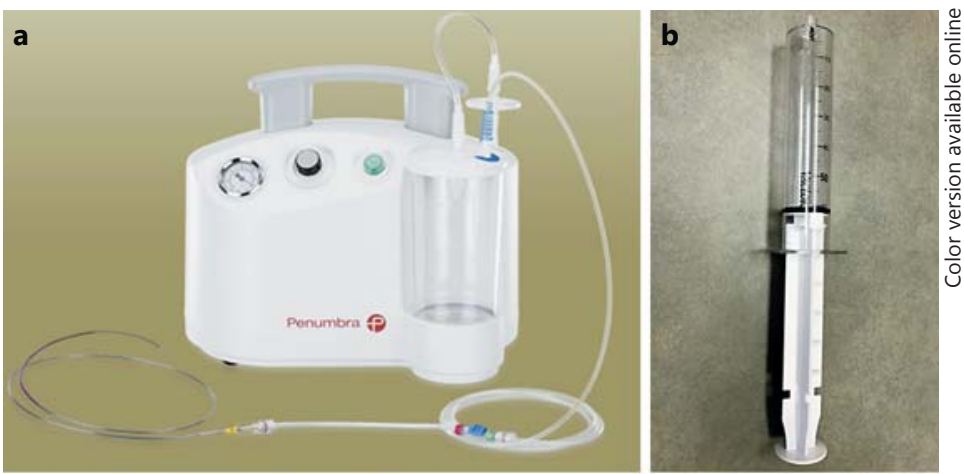

Fig. 1. a-c The three pumps tested were the Penumbra Max electrical pump (a), a 60-mL locking syringe (b), and the ASPIRE device (c). d The vacuum pressure testing apparatus consisted of a rotating hemostatic valve attached to 3/8-inch silicon tubing, which was connected to an oilfilled vacuum pressure gauge. The system was filled with water during all testing.
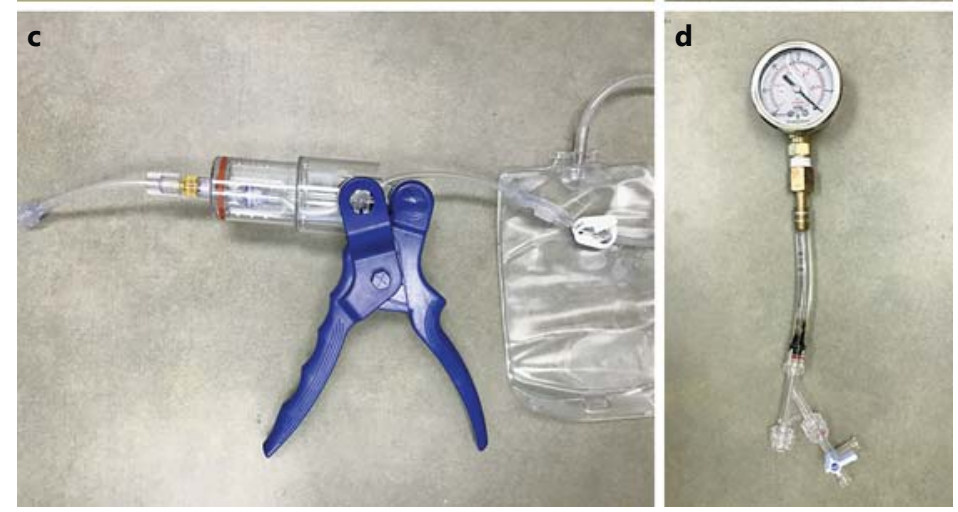

\section{Methods}

A variety of new-generation catheters were tested to evaluate the vacuum pressure transmitted to the tip of the catheter: the Penumbra (Alameda, CA, USA) Ace 064 (inner diameter $=1.63 \mathrm{~mm}$ ), the Stryker (Fremont, CA, USA) Catalyst6 (inner diameter $=1.52 \mathrm{~mm}$ ), the Medtronic (Dublin, Ireland) Arc 061 (inner diameter $=1.55 \mathrm{~mm}$ ), as well as the older Penumbra 041 aspiration catheter (inner diameter $=1.04 \mathrm{~mm}$ ). The Penumbra 041 was used as a comparator, given its smaller inner diameter compared to the other catheters.

Three different aspiration pumps were tested (Fig. 1a-c): the new-generation Penumbra Max electrical pump, a 60-mL locking syringe (Medallion; Merit Medical, Inc.), and the ASPIRE aspiration device (Control Medical Technology, LLC). Each device was used according to the manufacturer's instructions and to typical use. The Penumbra pump is an electrical pump with a collection canister and a control knob for vacuum pressure, which was turned to the highest setting. The ASPIRE device is a handheld pump that utilizes a barrel and plunger to create a vacuum when the handle is squeezed, with a one-way valve that allows the barrel to purge when the handle is released. Finally, the 60-mL locking syringe has a mechanism to hold the plunger against resistance (locking it in place) and was always tested at the maximum withdrawal position. However, in comparison to the other two devices, no mechanism exists to purge the syringe without releasing the vacuum. The Penumbra pump was attached to the catheter via the Penumbra aspiration tubing, the syringe was attached directly to the catheter via a Luer lock, and the ASPIRE device was attached to the catheter with the included Luer lock adapter tube.

The vacuum pressure testing apparatus (Fig. 1d) consisted of a rotating hemostatic valve (RHV) attached to 3/8-inch silicon tubing, which was connected to a DuraChoice 2-inch oil-filled vacuum pressure gauge (DuraChoice, Irving, TX, USA). The gauge has a range of 0 to -30 inches $\mathrm{Hg}$ (inHg). The system was filled with water during all testing, and the gauge was calibrated to atmospheric pressure.

Before each pressure test, the catheter was flushed and filled with water. The extension tubing of the aspiration source was filled with water if applicable. First, the aspiration source itself was directly connected to the pressure gauge apparatus, and the vacuum force was measured three times. The catheter was inserted into the RHV on the vacuum gauge system and locked in place. The aspiration source was then activated, and the gauge was monitored. Aspiration continued until the vacuum pressure remained static. For the Penumbra 
Table 1. Vacuum pressures measured at catheter tips (in inches $\mathrm{Hg}$ )

Froehler et al.: Comparison of Vacuum Pressures and Forces Generated by Different Catheters and Pumps for Aspiration Thrombectomy in Acute Ischemic Stroke

\begin{tabular}{|c|c|c|c|c|}
\hline & Sample 1 & Sample 2 & Sample 3 & Mean \\
\hline \multicolumn{5}{|l|}{ Penumbra pump } \\
\hline No catheter & 26 & 26 & 26 & 26.00 \\
\hline Catalyst6 & 26.5 & 25 & 26 & 25.83 \\
\hline Ace 064 & 25.5 & 26 & 26 & 25.83 \\
\hline Arc 061 & 25.5 & 25.5 & 26 & 25.67 \\
\hline Penumbra 041 & 25.5 & 25 & 25 & 25.17 \\
\hline \multicolumn{5}{|c|}{ 60-mL locking syringe } \\
\hline No catheter & 27 & 27 & 27 & 27.00 \\
\hline Catalyst6 & 27 & 28 & 27.5 & 27.50 \\
\hline Ace 064 & 25 & 27.5 & 27.5 & 26.67 \\
\hline Arc 061 & 27 & 27 & 27.5 & 27.17 \\
\hline Penumbra 041 & 26.5 & 27 & 27 & 26.83 \\
\hline \multicolumn{5}{|l|}{ ASPIRE device } \\
\hline No catheter & 27.5 & 27.5 & 28 & 27.67 \\
\hline Catalyst6 & 28 & 28 & 28 & 28.00 \\
\hline Ace 064 & 27.5 & 28 & 28 & 27.83 \\
\hline Arc 061 & 27.5 & 27.5 & 28 & 27.67 \\
\hline Penumbra 041 & 26.5 & 27 & 27 & 26.83 \\
\hline
\end{tabular}

pump, the valve on the extension tubing was opened to activate suction. The syringe was used by pulling the plunger back to the 60-mL mark and locking it in place. The ASPIRE device was used by pulling the handle repeatedly (typically 3-7 times) until the vacuum gauge remained static. Each measurement was repeated three times.

Once testing was completed with all of the catheters, a Rebar 18 (inner diameter $=0.021$ inches) was inserted into the catheter through an RHV. Both were filled with water and then inserted into the vacuum gauge apparatus in a coaxial manner. Aspiration testing was then repeated as above, the only difference being that the aspiration source was connected to the side arm of the RHV on the large-bore catheter to allow for the coaxial Rebar 18. Again, measurements were taken three separate times.

Finally, the theoretical maximum pulling force was mathematically calculated based on the actual vacuum pressure values and catheter sizes. Mean vacuum pressures were converted to Pascals and multiplied by the area of the catheter tip in $\mathrm{m}^{2}$ (using the manufacturer's reported inner diameter at the tip), yielding the theoretical pulling force in newtons $(\mathrm{N})$. The following tip areas for each of the catheters used were: Stryker Catalyst6, $1.81 \times 10^{-6} \mathrm{~m}^{2}$; Penumbra Ace 064, $2.08 \times 10^{-6} \mathrm{~m}^{2}$; Medtronic Arc 061, $1.88 \times$ $10^{-6} \mathrm{~m}^{2}$; and Penumbra 041, $8.49 \times 10^{-7} \mathrm{~m}^{2}$.

\section{Results}

There were 14 different catheter and aspiration source combinations and the vacuum pressure was measured three times each, for a total of 42 pressure measurements. The vacuum pressures measured at the catheter tips are shown in Table 1, and the mean pressures for each catheter and pump combination are shown in Figure 2. In general, there were no major differences in vacuum pressure between catheters or between aspiration pumps, with pressures ranging from -25 to -28 inHg. There was no difference between catheters, with mean vacuum pressures of $-27.11 \mathrm{inHg}$ for the Stryker Catalyst6, $-26.78 \mathrm{inHg}$ for the Penumbra Ace 064, -26.83 inHg for the Medtronic Arc 061, and -26.28 inHg for the Penumbra 041 aspiration catheter. Mean vacuum pressure across all catheters was $-25.63 \mathrm{inHg}$ for the Penumbra pump, -27.04 inHg for the syringe, and -27.58 inHg for the ASPIRE device. Differences between catheters and pumps were assessed by two-way ANOVA, which showed a 


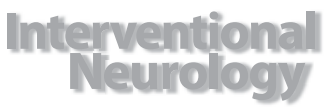

Table 2. Vacuum pressures with the Rebar 18 coaxial microcatheter (in inches $\mathrm{Hg}$ )
Intervent Neurol 2017;6:199-206

DOI: $10.1159 / 000475478$

2017 S. Karger AG, Basel www.karger.com/ine

Froehler et al.: Comparison of Vacuum Pressures and Forces Generated by Differen Catheters and Pumps for Aspiration Thrombectomy in Acute Ischemic Stroke

\begin{tabular}{|c|c|c|c|c|}
\hline & Sample 1 & Sample 2 & Sample 3 & Mean \\
\hline \multicolumn{5}{|l|}{ ASPIRE device } \\
\hline Catalyst6 + Rebar 18 & 26.5 & 27 & 26.5 & 26.67 \\
\hline Ace $064+$ Rebar 18 & 27 & 26.5 & 26.5 & 26.67 \\
\hline Arc $061+$ Rebar 18 & 26.5 & 26.5 & 26.5 & 26.50 \\
\hline Overall mean & & & & 26.61 \\
\hline \multicolumn{5}{|l|}{ Penumbra pump } \\
\hline Catalyst6 + Rebar 18 & 25 & 25 & 26.5 & 25.50 \\
\hline Ace $064+$ Rebar 18 & 23 & 25 & 24.5 & 24.17 \\
\hline Arc $061+$ Rebar 18 & 23.5 & 25 & 24 & 24.17 \\
\hline Overall mean & & & & 24.61 \\
\hline
\end{tabular}

Fig. 2. Mean vacuum pressures in inches $\mathrm{Hg}$ (inHg) measured at the catheter tip for each catheter and pump combination. The differences between pumps were statistically significant, but the differences between catheters were not (two-way ANOVA).

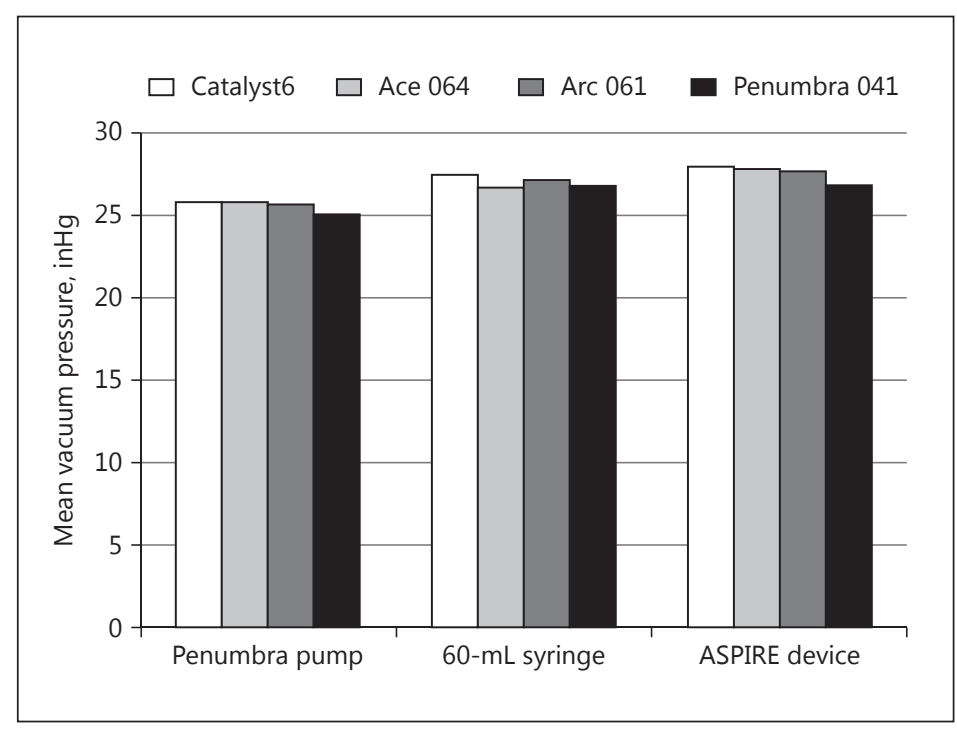

statistically significant difference between pumps $(p<0.001)$, but no significant difference between catheters $(p=0.105)$. Post hoc comparison between the mean vacuum pressures of all three pumps showed that it was significantly different (lower) in the Penumbra pump than in the syringe and in the ASPIRE device ( $p<0.01$; Tukey honest significant difference).

Catheters were then compared with the Rebar 18 microcatheter in place (Table 2), with the purposeful exclusion of the Penumbra 041 catheter and the $60-\mathrm{mL}$ syringe, as these would not be expected to be used in such a scenario in modern clinical practice. The mean vacuum pressure for the Penumbra Ace 064 when the microcatheter was within the aspiration catheter was $-24.61 \mathrm{inHg}$, and $-26.61 \mathrm{inHg}$ for the ASPIRE device $(p<0.0001$; Student $t$ test). Overall, the presence of the microcatheter resulted in a reduction in vacuum pressure of 1.2 inHg, a small but statistically significant difference $(p=0.005)$.

The theoretical clot pulling force was calculated for each pump and catheter based on measured vacuum pressure and catheter tip area. These calculated suction forces are shown in Figure 3. The mean suction forces for the catheters across all pumps were $0.167 \mathrm{~N}$ for the Stryker Catalyst6, $0.189 \mathrm{~N}$ for the Penumbra Ace 064, $0.171 \mathrm{~N}$ for the Medtronic Arc 061, and $0.0756 \mathrm{~N}$ for the Penumbra 041. The Penumbra Ace 064 catheter showed the highest potential force at the catheter tip, given its comparatively larger distal inner diameter, whereas the 
Fig. 3. Calculated theoretical pulling forces at the catheter tip in newtons (N). Force was calculated as the product of the vacuum pressure in Pascals multiplied by the inner area of the catheter tip in meters. This force reflects the theoretical clot extraction capability.

Froehler et al.: Comparison of Vacuum Pressures and Forces Generated by Different Catheters and Pumps for Aspiration Thrombectomy in Acute Ischemic Stroke

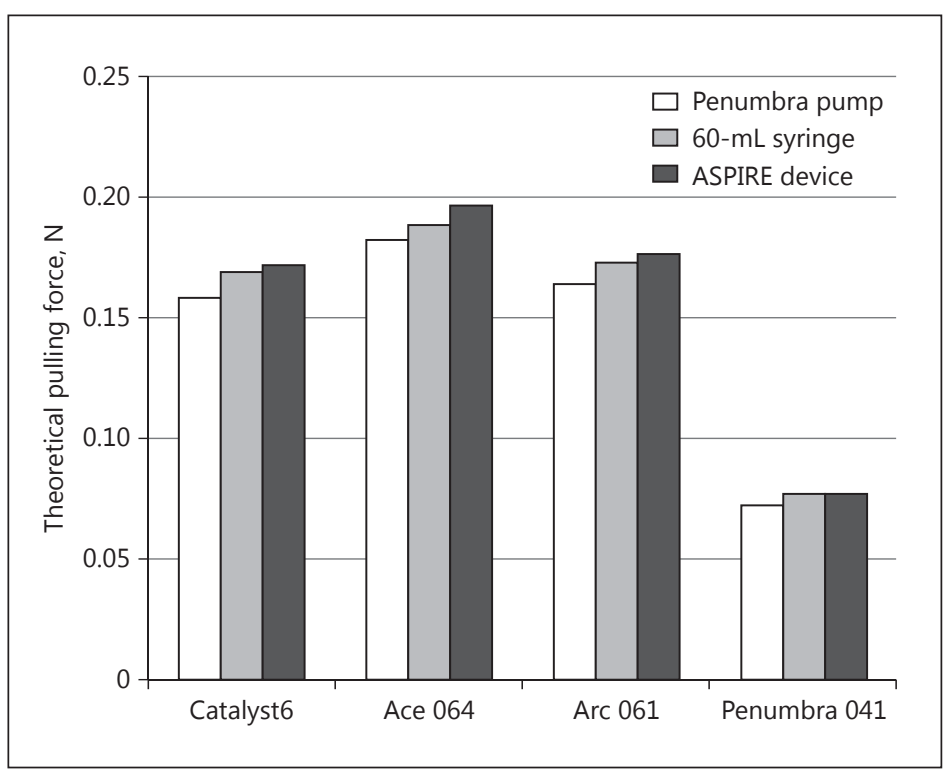

Penumbra 041 catheter has about half as much theoretical suction force, given its relatively small diameter. Suction forces were slightly higher for the ASPIRE device, given its modestly higher vacuum pressures as measured.

\section{Discussion}

This study showed that, in general, all catheters transmit roughly equivalent vacuum pressure, regardless of diameter. However, the suction force that is generated at the catheter tip is a product of the vacuum pressure and the catheter area, and larger catheters have a significant theoretical advantage in this regard.

There was not much difference in maximum vacuum pressure between the three pumps, all generating -26 to -28 inHg of vacuum. This value approaches the theoretical maximum for a vacuum, which is equal to the ambient atmospheric pressure (the normal barometric pressure at sea level is $29.92 \mathrm{inHg}$ ). There was a small but statistically significant difference between the pressure of the Penumbra pump and the other two, though this difference may not be functionally significant. Perhaps more important are factors that could allow decay of the vacuum. Specifically, when aspirating within a blood vessel, the system may start to fill with blood before the catheter tip becomes occluded by the clot. If that blood cannot be purged, then the absolute pressure of the vacuum generated will be reduced. The Penumbra pump has a collection canister for this purpose and is thus able to apply continuous suction. In this regard the syringe is a poor choice, as blood may enter the catheter and/or syringe and reduce the vacuum force. The ASPIRE device works like a syringe, but has a built-in valve that allows the chamber to purge when the handle is released. This allows the device to maintain a vacuum while purging so that additional aspiration can be performed.

While the transmitted vacuum pressures were similar between all catheters, the force calculations revealed more significant differences. It is this suction force which is most important in direct aspiration thrombectomy, and thus maximizing both the vacuum pressure and the catheter tip area can have a significant impact on the effectiveness of thrombectomy. Others have previously shown differences in aspiration flow rates between different catheters $[11,12]$, which is a function of the catheter radius to the fourth power. In contrast, force 
is the product of pressure and area, where the area of a circle is a function of the radius squared. So while the radius of the catheter does have less of an effect on force than it does on flow, it is still an exponential function, and thus larger catheters still offer the potential of better function.

We also showed that the presence of a microcatheter within the aspiration catheter has a minimal, but measurable, effect on the amount of vacuum pressure transmitted. However, as we have seen with the force calculations for individual catheters, the open area of the catheter tip has a substantial impact on the amount of suction force exerted on the clot. Therefore, one would expect that suction force would be significantly reduced if the microcatheter tip was allowed to protrude through the aspiration catheter tip. Importantly, if the microcatheter is pulled back to be just inside the catheter tip and not protrude through it, it should not have an impact on the suction area. In that scenario, suction force would only be minimally impacted by the slight reduction in transmitted vacuum pressure. Therefore, it would seem that the microcatheter does not need to be removed completely, as others have advocated with the "bare-wire thrombectomy" technique [19], but rather simply be pulled back so that the microcatheter tip is proximal to the aspiration catheter tip.

The results of these experiments suggest that the theoretical ideal thrombectomy conditions are created by a catheter with a large opening at the tip combined with a strong vacuum pump that will not decay, with the tip of the aspiration catheter beyond the coaxial microcatheter tip (if one is used). Among the catheters and pumps tested in these experiments, there was little difference in vacuum pressure generation or transmission. There were more significant differences in calculated suction force at the catheter tip, which is a function of catheter size. Since it is this suction force that is most relevant in direct aspiration thrombectomy, future aspiration catheters may be optimized with larger tip diameters.

\section{Acknowledgments}

The author is very grateful to Jeanne Froehler for assistance with data collection.

\section{Statement of Ethics}

As no human patients or animals were utilized in these experiments, no institutional ethical review was necessary.

\section{Disclosure Statement}

M.T. Froehler receives research funding from the NIH, Medtronic, Stryker, Penumbra, and Microvention, and serves as a consultant for Medtronic, Stryker, Control Medical Technology, and Blockade Medical.

\section{References}

1 Powers WJ, Derdeyn CP, Biller J, et al: 2015 American Heart Association/American Stroke Association Focused Update of the 2013 Guidelines for the Early Management of Patients With Acute Ischemic Stroke Regarding Endovascular Treatment: A guideline for healthcare professionals from the American Heart Association/ American Stroke Association. Stroke 2015;46:3020-3035.

2 Kuntze Soderqvist A, Andersson T, Ahmed N, Wahlgren N, Kaijser M: Thrombectomy in acute ischemic stroke: estimations of increasing demands. J Neurointerv Surg 2016, Epub ahead of print.

3 Walcott BP, Boehm KM, Stapleton CJ, Mehta BP, Nahed BV, Ogilvy CS: Retrievable stent thrombectomy in the treatment of acute ischemic stroke: analysis of a revolutionizing treatment technique. J Clin Neurosci 2013; 20:1346-1349. 
Froehler et al.: Comparison of Vacuum Pressures and Forces Generated by Different Catheters and Pumps for Aspiration Thrombectomy in Acute Ischemic Stroke

4 Turk AS, Frei D, Fiorella D, et al: ADAPT FAST study: a direct aspiration first pass technique for acute stroke thrombectomy. J Neurointerv Surg 2014;6:260-264.

5 Wallace AN, Kansagra AP, McEachern J, Moran CJ, Cross DT 3rd, Derdeyn CP: Evolution of endovascular stroke therapies and devices. Expert Rev Med Devices 2016;13:263-270.

6 Turk AS, Spiotta A, Frei D, et al: Initial clinical experience with the ADAPT technique: a direct aspiration first pass technique for stroke thrombectomy. J Neurointerv Surg 2014;6:231-237.

7 Kang DH, Hwang YH, Kim YS, Park J, Kwon O, Jung C: Direct thrombus retrieval using the reperfusion catheter of the Penumbra system: forced-suction thrombectomy in acute ischemic stroke. AJNR Am J Neuroradiol 2011;32:283-287.

8 Delgado Almandoz JE, Kayan Y, Young ML, et al: Comparison of clinical outcomes in patients with acute ischemic strokes treated with mechanical thrombectomy using either Solumbra or ADAPT techniques. J Neurointerv Surg 2016;8:1123-1128.

9 Campbell BC, Donnan GA, Lees KR, et al: Endovascular stent thrombectomy: the new standard of care for large vessel ischaemic stroke. Lancet Neurol 2015;14:846-854.

10 Vargas J, Spiotta A, Fargen K, Turner R, Chaudry I, Turk A: Long term experience using the ADAPT technique for the treatment of acute ischemic stroke. J Neurointerv Surg 2017;9:437-441.

$11 \mathrm{Hu}$ YC, Stiefel MF: Force and aspiration analysis of the ADAPT technique in acute ischemic stroke treatment. J Neurointerv Surg 2016;8:244-246.

12 Simon SD, Grey CP: Hydrodynamic comparison of the Penumbra system and commonly available syringes in forced-suction thrombectomy. J Neurointerv Surg 2014;6:205-211.

13 Penumbra Pivotal Stroke Trial Investigators: The Penumbra Pivotal Stroke Trial: safety and effectiveness of a new generation of mechanical devices for clot removal in intracranial large vessel occlusive disease. Stroke 2009;40:2761-2768.

14 Jankowitz B, Aghaebrahim A, Zirra A, et al: Manual aspiration thrombectomy: adjunctive endovascular recanalization technique in acute stroke interventions. Stroke 2012;43:1408-1411.

15 Jankowitz B, Grandhi R, Horev A, et al: Primary manual aspiration thrombectomy (MAT) for acute ischemic stroke: safety, feasibility and outcomes in 112 consecutive patients. J Neurointerv Surg 2015;7:27-31.

16 Fojtik SP, Kronick LS: Cardiovascular innovations: novel mechanical aspiration system to improve thrombus aspiration speed, force, and control. Cardiovasc Revasc Med 2013;14:160-163.

17 Humphries W, Hoit D, Doss VT, et al: Distal aspiration with retrievable stent assisted thrombectomy for the treatment of acute ischemic stroke. J Neurointerv Surg 2015;7:90-94.

18 Massari F, Henninger N, Lozano JD, et al: ARTS (Aspiration-Retriever Technique for Stroke): initial clinical experience. Interv Neuroradiol 2016;22:325-332.

19 Nikoubashman 0, Alt JP, Nikoubashman A, et al: Optimizing endovascular stroke treatment: removing the microcatheter before clot retrieval with stent-retrievers increases aspiration flow. J Neurointerv Surg 2017; 9:459-462. 